\title{
The Design and Implementation of Cloud Storage System Based on Hiphop-php
}

\author{
Chenhe Zhu ${ }^{[1]}$;Zhiyi Fang ${ }^{[2]}$;Yao Sun ${ }^{[3]}$;Zheng Fang ${ }^{[4]}$;Fanfan Zhang ${ }^{[5]}$; Jiliang Wang $^{[6]}$ \\ Computer Science and Technology. JiLin University, Changchun China \\ Email: syloyy@163.com
}

\begin{abstract}
With the rapid development of Internet technology, the data of the users' information have raised up largely, so internet storage became more and more important in today's life. In this paper, a cloud storage system has been designed and implemented. By using Hiphop-php technology, the performance of cloud storage system has been improved.
\end{abstract}

\section{Keywords-cloud storage; Hiphop-php}

\section{INTRODUCTION}

With the rapid development of Internet and communication technology, and the volume of explosive growth of user information data, so the traditional technical architecture is already unable to meet the needs of users and enterprises for data storage ${ }^{[1]}$. Cloud storage is becoming provide portable data storage service for ordinary users, which is also a first choice for the enterprise to solve the requirements of the data capacity, management, and the big cost of operational .The cloud storage system which is described in this article is a cloud storage application, that is based on third party ability of open platform development, which is mainly to provide network disk service for users, and for the users to store data files. Through the application server to interact with the user, by calling the platform interface makes the user files which are stored on third-party platform server, this is a typical type of cloud service outsourcing. ${ }^{[2,3]}$

In this article, we use the Hiphop-php technology to develop a cloud storage based on B/S application, calling the storage capacity of the third party server. The main function of Hiphop-php program is to convert PHP script to $c^{++}$code, so that the WEB server load pressure can be greatly reducing. ${ }^{[4,5]}$

\section{THE CURRENT RESEARCH STATUS OF CLOUD STORAGE SYSTEM}

Cloud storage arouses everybody's enthusiasm and ushered in the rapid market growth as soon as that enter China, that has emerged a large number of cloud storage application in just a few short years, such as Huawei, 360, kinsman has launched its own cloud storage applications. Some enterprises based on the existing user base, establishing and developing their own cloud storage applications, some enterprises halfway decent, with his bare hands to build his own cloud storage system, the reason is only one: they all look good which cloud storage market ${ }^{[6]}$. At same time ,the user are also showing an unprecedented support and enthusiasm in cloud storage.115network location announced their registered users has exceeded 30 million, while Huawei's network location also announced its own registered users has exceeded 20 million, which not only reflect the cloud storage application of high-speed development, comparing with the amount of Internet users, 420 million Internet users, this is to remind us, the cloud storage market of our country still has a considerable development space. However, large user base of cloud storage system performance and load problem poses a challenge. ${ }^{[7,8]}$

Open business capacity platform is a server platform based on PaaS (Platform as a service) business model. Currently, many cloud storage services are realized through outsourcing, cloud storage service operators do not need to build their own file storage system, they can use the interfaces provided by platform to outsource the data store tasks. Some of these platforms are focus on cloud storage, some have much business and cloud storage is a part of them. What the well-known cloud storage DropBox used is Amazon S3 platform. There are two operators in this kind of cloud service, the cloud service operator and the data storage operator. The cloud service operator communicates with the users and manages user's information while the latter try their best to manage the users' files on the platform. Users didn't feel the data storage operators but they stay with them all day long. With the development of mobile Internet, developing the open business capacity platform applies to mobile Internet is on the time. The platform can not only provides cloud service but also provides telecommunications business capacity and other business capacities.

\section{THE DESIGN AND IMPLEMENTATION OF CLOUD} STORAGE SYSTEM

\section{A. System function request}

1) Registration and Login part

- Registration: System statistics basic user information, then send the confirmation mail (SMS) to mailbox (mobile phone), and user can verify messages (SMS) to invoke.

- Login: Users login according to the input verification code, user ID and other login information with user interface friendly. The password stored in the local database in the form of ciphertext.

2) File management part 
- Add/delete directory (folder): Users can create the new folder in the user folder which will be automatic naming. Same path folder will not name. And the user can choose to delete the folder. All the contents of folder will be deleted.

- Rename: renaming the folder or file name, but files (folders) name in the same path should be different.

- File upload: when uploading the specified file to the current folder, it is selected by the user if encrypting the file. Files which need to be encrypted will be stored to platform server after encryption.

- File download: Display all files in the user profiles. Download the specified file by the browser.

- File encryption: Encrypt user files in the application server. The key can be provided by the user and can also be generated by the system default.

- Delete file: file delete operation will put the specified files in the recycle bin. And the file in the recycle bin can be restored; Users can choose files in the recycle bin to delete thoroughly. Deleting the file in the recycle bin cannot be resumed. Recycling station file still occupied user file capacity.

- $\quad$ File transfer: It will transfer the specified files from the current path to the specified path. The user can freely move the file position.

- The Recycle Bin: Recycle bin is for storing the files which users delete, and provide remove the file operation. Removing files will be deleted thoroughly. Recycle bin provides quick clear recycle: "Empty the recycle bin" function can delete all files from the recycle station.

- Capacity management: Statistics the file size uploading by the user to gain the total used capacity. And the total capacity which could be used will be distributed when users registered. There is a reminder to be sent to the user when the total used capacity is more than it.

\section{B. Performance design}

Through systematic analysis of cloud storage application server, the big part of occupation of the server operation performance is complex generalpurpose computing part of PHP (encryption, decryption, other computing algorithm). Since the PHP is a kind of script language, the use of memory and CPU ratio of this type language is very low. So the best way to improve the performance of system is to use $\mathrm{C} / \mathrm{C}++$ instead of PHP language for calculating part. So this article uses the technology of Hiphop for system optimization.

The Hiphop-php technology is developed by Facebook, using these technology development personnel can convert PHP key operation section to $\mathrm{C}++$ code rapidly. This way can avoid a lot of manual operation and decrease the possibility of error of the conversion program. The $\mathrm{C}++$ language memory management is more powerful than PHP, generally the operations for some memory garbage collection and other commonly used memory management are directly running in the physical machine operation, instead of running in the PHP interpreter. So the use of Hiphop-php technology will take less resource than the direct use of PHP. On the other hand, every time a user request to the server, the PHP interpreter will request explanation once for each. So if the number of users increases, the efficiency of the server will descend straightly; but with the application of the Hiphop technology, the work of the WEB program interpretation has been handed over to the compiler, for each user request, background processing program will use the binaries and response operation that has been compiled to improve the performance of the system.

\section{System architecture design}

The module of system file operation uploads, downloads and deletes data by call to the interface of open service platform for cloud storage, and the platform provides Restful Web Service interface. When you register at the platform, you will get AppKey and AppSecret then you will receive an authentication named token, finally, authorization is done. These systems make a simple encapsulation to the platform interface, and provide a test of PHP SDK. The cloud storage interface uploads files by means of PUT, and sends a ask for filedownload to platform server by means of GET, then server return the link of file-download, and sends a ask for file-delete to the platform server by means of GET to delete files, finally server will return a corresponding result.

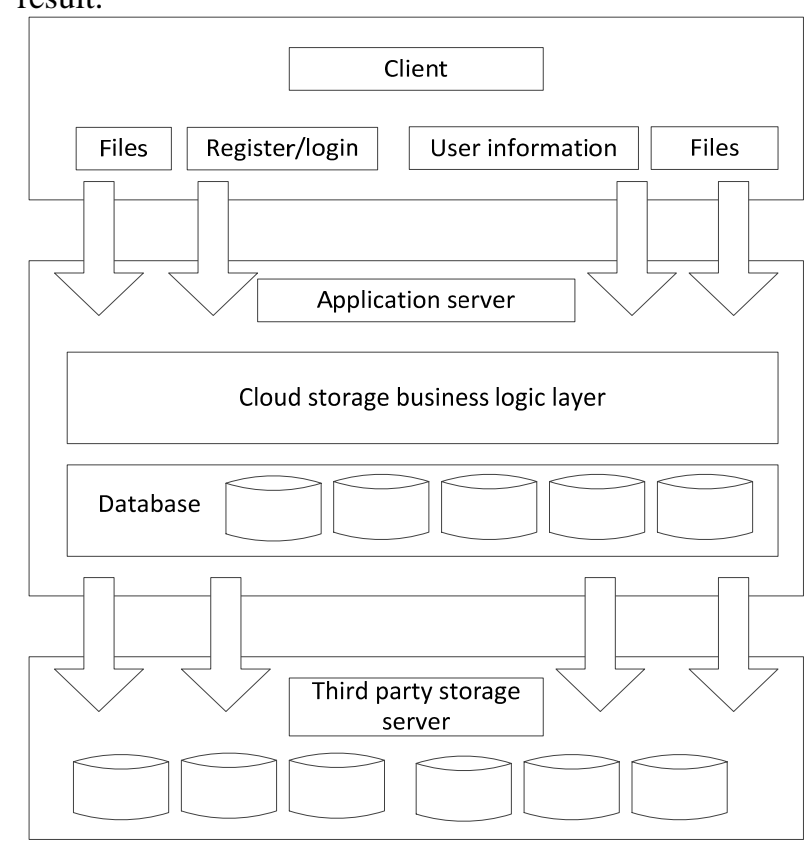

Figure 1 Architecture of cloud system 
Firstly, user file is renamed at application server and save the information of file then it will be encrypted. The next step is to upload file to the platform buy means of call to cloud storage interface, and save it whose name is unique at the whole situation and in all the file of all users. The system makes a filename by user id, uploading time and the information of some files, to guarantee that the name is unique. At application server, server organizes the file structure of users by the information which is saved as uploading, such as user name, initial filename and the file path. For example, it will distinguish the owner of this file by the name of the user who uploaded this file. Judging which file dose the file belongs to by the file path, deciding the file name which appear in the former interface by initial file name.

\section{Module design}

The system mainly contains four modules: Register module, user information management module, file operations module and manage module.

- Register module: this module includes the user name check; send authentication information; account activation and user information initialization.

- User information management module: this module includes user information collected and modify; password change. Users will fill in some basic information when they were registering and they can improve their information after successful registration. User can change password and the system will send an email to user's mailbox.

- File operations module: this module includes basic file operations such as files uploading and downloading, files moving, files recycle and directory operations.

- Manage module: this module is used to manage users' information, files information and users permissions.

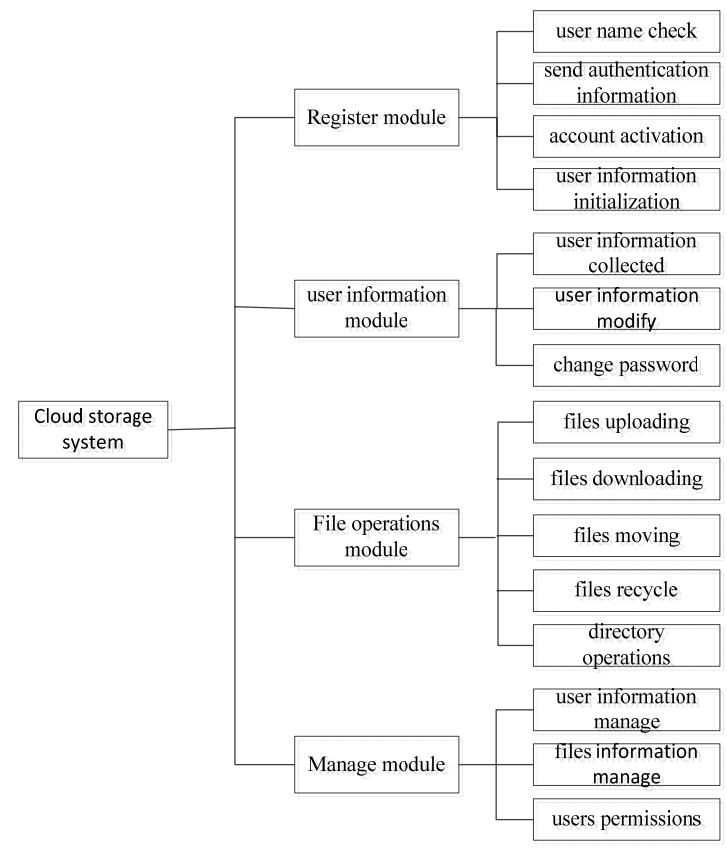

Figure 2 system module design

\section{E. System implementation}

The user homepage is the most important interface. It can divide into The Page Header District, The Page Side District and The File Show District. The Page Header District provides a switch among the different modules. The Page Show District is used to show user files. The File Side District provides some operations to file or file path, such as building file, putting into the recycle bin and so on. The right key reflects the most operations to files, such as shearing, deleting. Figure 3 shows the accomplishment of main system interface of PC terminal.

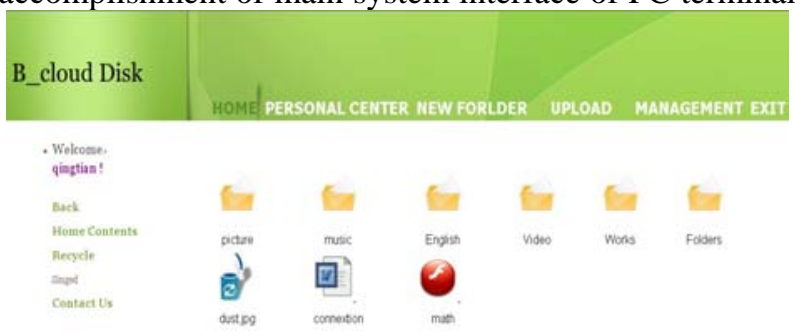

Figure 3 the homepage of cloud storage system

\section{TEST}

To confirm the property of this system, we test the system with software named Apache HTTP server benchmarking tool which is exploited by Apache Software Foundation in this paper. 


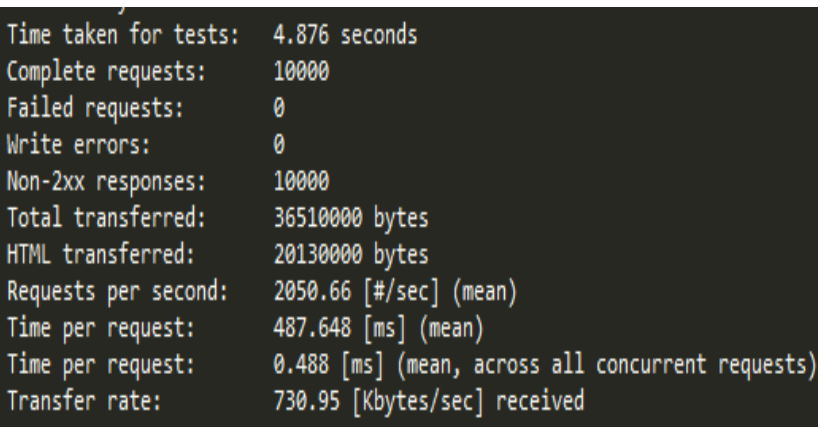

Figure 4 Result of the test

Now let's introduce our testing measure: for the cloud storage system, we suppose that there are 1000 users to make user of this system at the same time, then we make 10000 tests in a row. This is the testing command:

"ab -n10000 -c1000 http://serverdomain"

The serverdomain is the address of server. For example:

We can figure out that there is no failure from the testing result, and the average waiting time is just 487.648 milliseconds. The concurrent ability of the whole system is fit for our design requirement.

\section{SUMMARIES}

With the rapid development of Internet technology, the data of the users' information have raised up largely. It makes cloud storage render a portable data storage service to domestic consumers gradually. The article is by calling the third party storage server, use the php to design and implement a cloud storage system. In addition, we use the Hiphop to optimize the memory management and thread management of the system, from this, make the whole system have a stabilized concurrency ability and better performance.

\section{REFERENCES}

[1]. C.Wang,Q.Wang,K.Ren and W.Lou. "Ensuring data storage security in cloud computing” in Proc. Of IWQoS’09, Charleston, South Carolina, USA, 2009.

[2]. Paul Biggar, Edsko de Vries, David Gregg, A practical solution for scripting language compilers, Proceedings of the 2009 ACM symposium on Applied Computing, March 08-12, 2009

[3]. Gregor Richards , Christian Hammer , Brian Burg , Jan Vitek, The eval that men do: A large-scale study of the use of eval in javascript applications, Proceedings of the 25th European conference on Object-oriented programming, July 25-29, 2011.

[4]. G. Ateniese, R. Burns, R. Curtmola, J. H. amd Lea Kissner, Z. Peterson, and D. Song. Provable data possession at untrusted stores. In Proceedings of the ACM Conference on Computer and Communications Security, 2007

[5]. R. Curtmola, O. Khan, R. Burns, and G. Ateniese. MR-PDP: Multiple-replica provable data possession. In Proceedings of the International Conference on Distributed Computing Systems, 2008.

[6]. Fang Hao , T. V. Lakshman , Sarit Mukherjee , Haoyu Song, Secure cloud computing with a virtualized network infrastructure, Proceedings of the 2nd USENIX conference on Hot topics in cloud computing, pp.16-16, June 22-25, 2010,

[7]. Timothy Wood , Alexandre Gerber , K. K. Ramakrishnan , Prashant Shenoy, Jacobus Van der Merwe, The case for enterprise-ready virtual private clouds, Proceedings of the 2009 conference on Hot topics in cloud computing, June 15, 2009, San Diego, California.

[8]. Michael Zink , Kyoungwon Suh , Yu Gu , Jim Kurose, Characteristics of YouTube network traffic at a campus network Measurements, models, and implications, Computer Networks: The International Journal of Computer and Telecommunications Networking, v.53 n.4, p.501-514, March, 2009. 